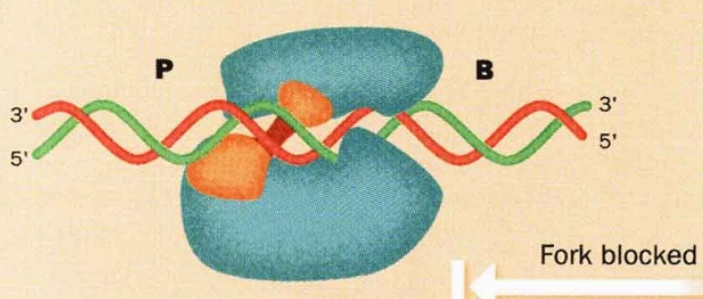

Fork passes through

FIG. 2 Model of the Tus (protein)-Ter (DNA) complex, devised by Kamada et $\mathrm{al}^{3}{ }^{3}$, in which the DNA is shown to extend beyond the contact region at each end. A replication fork moving from right to left (arrow) is arrested at the B (blocking) end. But a fork moving in the opposite direction is not impeded upon encountering the $P$ (passage) end and passes through the complex freely.

to each $\mathrm{Ter}$ site as a monomer ${ }^{7,8}$. Within the Tus-Ter complex (Fig. 2) the protein is organized into two domains, each composed of $\alpha$-helical (blue) and $\beta$-sheet (yellow) portions, connected by two antiparallel $\beta$-strands (brown). This creates a large, positively charged central cleft into which double-stranded DNA fits, such that the DNA sits across the interdomain region, and is flanked by the two large protein domains. The DNA deviates significantly from the Watson-Crick B-form the DNA is underwound, and the backbone is deformed in regions where the DNA makes particularly close contact with the protein. Altogether, the protein makes polar contacts with more than two-thirds of the phosphates in the DNA-binding region. The interdomain $\beta$-region holds the DNA in a vice-like grip, penetrating the deepened major groove to varying extents, and making intimate contacts with several bases therein. So this $\beta$-region is responsible for recognition of the Ter sequence, as well as for tight binding by Tus. Notably, the tight-binding region is asymmetrically positioned towards the left extremity of the complex, as shown in Fig. 2.

A replication fork moving from right to left is arrested upon encountering the B (blocking) end of the complex. The extent to which Tus grasps and surrounds the DNA enables its rightmost face to present a physical barrier to the helicase. This means that the helicase cannot disrupt the tight Tus-Ter contacts. When moving from left to right, towards the P (passage) end, the helicase encounters no barrier, and proceeds to strip off the relatively exposed red strand and disrupt the Tus primary binding site. Furthermore, the unwinding motion of the helicase could relax the hold of Tus on the DNA by levering back its flanking domains and thereby causing its displacement. This model is given more credence by the fact that 13 Tus mutants which lowered or abolished fork arrest in vivo showed reduced, or no, Ter binding. Most of the mutations were mapped to the interdomain region involved in DNA binding.

So Kamada et $a l^{3}$ see Tus as being essentially a polar clamp on the DNA. A possible molecular basis for such a clamp has been described ${ }^{9}$ in terms of a stable barrier to unwinding at the blocking end because of more extensive crosslinking between Tus and the two DNA strands at that end. But the Kamada model is crucially different - the blocking end of Tus can prevent the helicase from reaching the tight-binding region because Tus protrudes beyond this region, and because of its configuration around the DNA.

The observed structure itself does not rule out the possibility of interactions between Tus and the helicase or some other protein involved in the replication process, and the model won't necessarily satisfy the proponents of the requirement for such an interaction. However, the crystal structure will enable them to test their views critically through detailed probing of the surface features that are required at the blocking end of Tus for fork arrest.

How might these findings apply to the $B$. subtilis replication terminator protein (RTP)-Ter system? Although the Kamada model would certainly strengthen a case against the involvement of specific RTP-helicase interactions in fork arrest, it is possible that the situation in B. subtilis is quite different from that in $E$. coli. Tus and RTP have no sequence similarity, and neither do the DNA terminators of the two organisms. Also, the functional $B$. subtilis arrest complex requires an interaction between two RTP dimers bound to a larger terminator ${ }^{10}$. But the $E$. coli picture does raise the possibility of a similarly configured steric barrier arising from the apparently more intricate situation in B. subtilis.

R. G. Wake is in the Department of Biochemistry, University of Sydney, New South Wales 2006, Australia.

\footnotetext{
1. Hill, T. M. in Escherichia coli and Salmonella 2nd edn (eds Neidhardt, F. C. et al.) 1602-1614 (ASM, (eds Neidhardt, F. C. et
Washington DC, 1996).

2. Hill, T. M. Annu. Rev. Microbiol. 42, 603-633 (1992).

3. Kamada, K., Horiuchi, T., Ohsumi, K., Shimamoto, N. \& Morikawa, K. Nature 383, 598-603 (1996).

4. Lee, E. H., Kornberg, A., Hidaka, M., Kobayashi, T. \& Horiuchi, T. Proc. Natl Acad. Sci. USA 86, 9104-9108 (1989).

Khatri, G. S., MacAllister, T., Sista, P. R. \& Bastia, D. Cell 59, 667-674 (1989).

6. Hiasa, H. \& Marians, K. J. J. Biol. Chem. 267, 11379-11385 (1992).

. Sista, P. R., Hutchison, C. A. \& Bastia, D. Genes Dev. 5, 74-82 (1991)

8. Coskun-Ari, F. F., Skokotas, A., Moe, G. R. \& Hill, T. M. J. Biol. Chem. 269, 4027-4034 (1994)

9. Gottlieb, P. A. et al. J. Biol. Chem. 267, 7434-7443 (1992).

10. Langley, D. B., Smith, M. T., Lewis, P. \& Wake, R. G. Mol.
} Microbiol. 10, 771-779 (1993).

\section{Deeply deluded}

WE see things in three dimensions because our two eyes have a useful horizontal separation. Each eye sees nearby objects in a different position against the background of more distant ones; the brain compares the two images and derives a view with depth.

Random-dot and repeat-unit stereograms, those popular illusions in many books and posters, subtly exploit this mechanism. They have a pattern of many slightly different units repeated across the page, designed to subvert the eyes' visual fusion of the image. The right eye may focus on unit $n$ while the left eye focuses on the very similar unit $n+1$.

This misfusion then propagates across the whole stereogram. The units are shaped and positioned so that this false correlation gives a consistent impression of a scene in depth. Prolonged staring is often needed to see the effect.

The illusion would be more immediate if the eyes could be prevented from making their initial, correct matching between the two views. So Daedalus is doing it. He points out that a diffraction grating appears a different colour from different directions (look at a credit-card hologram). He is devising a computercontrolled holographic laser-printing engine to generate stereograms with diffractive repeat units. Left and right eyes will view each unit from a different angle, and see it in a different colour. The left and right images will be taken to arise from different objects, and will fail to fuse. Instead, fusion will occur between adjacent units, designed to look the same colour to left and right eyes. Prolonged staring will not be needed: the depth illusion will spring to the eye, convincingly and at once.

To defray the expense of the research, the first applications will be military. DREADCO's stereographic camouflage will make tanks and guns look much farther away than they really are, or give them the depth outlines of trees or buildings. Commercial products will soon follow. Stereographic clothes will augment (or diminish) desired regions of the wearer's figure; stereographic wallpaper will give the tiniest flat the illusion of spacious grandeur.

But nature may have got there first. Many animals have vertical stripes or other units which repeat horizontally, but not quite exactly. Such striking patterns, says Daedalus, cannot conceal the creature; they must camouflage it stereographically. He is now wandering round the local zoo, gazing abstractedly at zebras and tigers and angelfish, waiting for them to seem suddenly more distant, or to dissolve into a pattern of unconnected shapes. 